\title{
Use of a T-cell interferon- $\gamma$ release assay for the diagnosis of tuberculous pleurisy
}

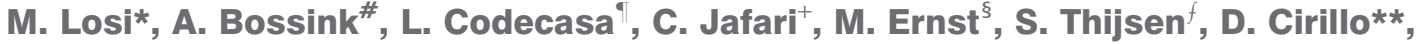 \\ M. Ferrarese ${ }^{\top}$, U. Greinert ${ }^{+}$, L.M. Fabbri*, L. Richeldi* and C. Lange ${ }^{+}$for the \\ European Tuberculosis Network TBNET
}

ABSTRACT: The diagnosis of pleural tuberculosis (pITB) by the analysis of pleural effusions (PEs) with standard diagnostic tools is difficult. In routine clinical practice, the present authors evaluated the performance of a commercially available Mycobacterium tuberculosis (MTB)specific enzyme-linked immunospot assay on peripheral blood mononuclear cells (PBMCs) and pleural effusion mononuclear cells (PEMCs) in patients with suspect pITB.

The T-SPOT.TB test (Oxford Immunotec Ltd, Abingdon, UK) was performed on PBMCs and PEMCs in 20 patients with a clinical and radiological suspect of pITB and in 21 control subjects with a diagnosis of PE of nontuberculous origin at four centres participating in the European Tuberculosis Network.

In total, $18(90 \%)$ out of 20 patients with pITB tested T-SPOT.TB-positive on PBMCs and 19 (95\%) out of 20 on PEMCs. Among controls, T-SPOT.TB was positive in seven out of 21 (33\%) patients when performed on PBMCs (these patients were assumed to be latently infected with MTB) and five (23\%) out of 21 when performed on PEMCs. Sensitivity and specificity of T-SPOT.TB for the diagnosis of active pITB when performed on PEMCs were 95 and $76 \%$, respectively.

Enumerating Mycobacterium tuberculosis-specific T-cells in pleural effusion mononuclear cells by ELISPOT is feasible in routine clinical practice and may be useful for a rapid and accurate diagnosis of pleural tuberculosis.

KEYWORDS: Culture filtrate protein-10, early secretory antigenic target-6, pleurisy, T-cell interferon- $\gamma$ release assay, tuberculosis

$\mathbf{T}$ uberculosis (TB) remains a major cause of morbidity and mortality, and represents the most frequent cause of death by a single infectious agent worldwide [1]. Pleural TB (plTB) is the second most common extrapulmonary manifestation of active Mycobacterium tuberculosis (MTB) infection after lymph node TB, accounting for up to $23 \%$ of TB cases [2] and $\sim 30 \%$ of pleural effusions (PEs) in Western Europe [3].

Pleural biopsy for culture of MTB, MTB nucleic acid amplification and histopathological detection of caseating granulomas are regarded as the gold standard for the diagnosis of plTB, with sensitivities of 39-80, 90 and 50-97\%, respectively [4-12].

The sensitivity for the detection of active TB infection is higher in pleural biopsies compared with pleural fluid, although the procedure is invasive and is associated with more clinical complications. Pleural taps and direct diagnosis of plTB from PEs would be preferred in the clinical setting.

However, culture of MTB, detection of MTBDNA and detection of alcohol-acid fast bacilli from PEs showed sensitivity for plTB ranging from $12-70 \%$ (with the majority of studies showing a sensitivity $<30 \%$ ), $30-100 \%$ (in culture-negative cases $30-60 \%$ ) and $<10 \%$, respectively $[4,8,10,12-14]$.

Recently, MTB-specific T-cell interferon (IFN)- $\gamma$ release assays (TIGRAs) have been developed as an enzyme-linked immunospot assay (ELISPOT) and as an enzyme-linked immunosorbent assay (ELISA) for the diagnosis of MTB infection from the peripheral blood [15]. These assays use antigens, early secretory antigenic target (ESAT)-6 and culture filtrate protein (CFP)-10, encoded in the genomic region of difference (RD)-1 of MTB which are absent in most nontuberculous mycobacteria, including the vaccination strains of Mycobacterium bovis bacille
AFFILIATIONS

*Section of Respiratory Disease, Dept of Oncology and Hematology, University of Modena and Reggio Emilia, Modena,

'TB Reg. Ref. Centre, Villa Marelli, Niguarda Hospital,

**Emerging Bacterial Pathogen Unit, San Raffaele Hospital, Milan, Italy,

${ }^{*}$ Depts of Pulmonary Diseases and Tuberculosis and

${ }^{f}$ Medical Microbiology and Immunology, Diakonessenhuis Utrecht, Utrecht, The Netherlands, +Division of Clinical Infectious Diseases, and

${ }^{\S}$ Immune-cell Analytics, Research Center Borstel, Borstel, Germany.

CORRESPONDENCE

C. Lange

Division of Clinical Infectious

Diseases

Medical Clinic

Research Center Borstel

Borstel

Germany

Fax: 494537188313

E-mail: clange@zz-borstel.de

Received:

June 052007

Accepted after revision:

August 012007

SUPPORT STATEMENT

C. Lange is supported by the H. W. \&

J. Hector Foundation (Mannheim,

Germany).

STATEMENT OF INTEREST

A statement of interest for L.M.

Fabbri can be found at

www.erj.ersjournals.com/misc/

statements.shtml

European Respiratory Journal

Print ISSN 0903-1936

Online ISSN 1399-3003 
Calmette-Guerin [16]. For the diagnosis of active pulmonary TB and latent TB infection, TIGRAs are more specific and probably more sensitive than the tuberculin skin test (TST) [15-20].

As only a small minority of lymphocytes of the human body circulate in the blood [21] neither immunological test can accurately distinguish between untreated active TB, latent TB infection (LTBI) or treated active TB when TIGRAs are performed on blood cells [17, 22].

However, in active TB, MTB-specific T-cells clonally expand and are recruited to the site of the infection $[23,24]$. Therefore, enumerating effector T-cells by ELISPOT at the site of the infection should enable more specific diagnosis of active TB than enumerating effector T-cells in the blood alone [25]. Recently, a much higher concentration of ESAT-6-specific IFN$\gamma$ producing effector T-cells was observed in pleural effusions of plTB patients compared with peripheral blood, while MTBspecific T-cells were not detected in pleural effusions of patients with nontuberculous causes of pleurisy [26]. The study by WILKINSON et al. [26] was performed using the Lalvani ELISPOT assay, in which CFP-10 antigen was not tested and the case-control study design did not allow an evaluation of the performance of this approach for diagnosis of plTB in routine clinical practice.

Therefore, the present authors prospectively determined to evaluate the performance of the commercially available MTBspecific ELISPOT (T-SPOT.TB; Oxford Immunotech Ltd, Abingdon, UK) using both ESAT-6 and CFP-10 antigens for the diagnosis of plTB in routine clinical practice at several centres within the European Tuberculosis Network (TBNET).

\section{MATERIALS AND METHODS \\ Patients}

Following informed consent and ethic committee approval, patients with one-sided exudative pleural effusions and a medical history compatible with plTB presenting to the Dept of Respiratory Medicine at the University Hospital of Modena and Reggio-Emilia (Modena, Italy); the Villa-Marelli Institute (Milan, Italy); the Medical Clinic of the Research Center Borstel (Borstel, Germany); and the Dept of Respiratory Medicine and Tuberculosis at the Diakonessenhuis (Utrecht, The Netherlands), between January 2005 and November 2006 were enrolled in the study. Peripheral blood mononuclear cells (PBMC) were obtained by a venous blood draw of $30 \mathrm{~mL}$ for MTB-specific ELISPOT (T-SPOT.TB). Pleural tap was performed according to standard procedures [27] and was sent for microbiological culture, MTB-specific DNA amplification, routine biochemical examination and MTB-specific ELISPOT.

\section{Definition of cases, presumptive cases and controls}

Patients had a definitive diagnosis of plTB if MTB was cultured from pleural fluid or pleural biopsy, or if the result of the MTBspecific DNA amplification from the pleural fluid or pleural biopsy was positive. Patients had a presumptive diagnosis of plTB if MTB could not be cultured from the pleural fluid or pleural biopsy and the result of the MTB-specific DNA amplification from the pleural fluid or pleural biopsy was negative; these patients had to have a positive treatment response to a full course of anti-tuberculous therapy, and had to have no alternative diagnosis of pleurisy other than TB.
Patients were defined as having no plTB (controls) if: an alternative clinical diagnosis of pleurisy was established; MTB could not be cultured from the pleural fluid or pleural biopsy; the result of the MTB-specific DNA amplification from the pleural fluid or pleural biopsy was negative; patients were not treated for $\mathrm{TB}$; and patients did not develop signs or symptoms of TB after 3 months.

\section{ELISPOT assay}

MTB-specific ELISPOT assays were performed using testplates from the T-SPOT.TB test [28-30]. PE mononuclear cells (PEMCs) were prepared by Ficoll-Hypaque gradient centrifugation from PEs. Briefly, 250,000 PBMCs or 250,000 mononuclear cells from the PEMCs were plated overnight on 96-well plates that had been pre-coated with a mouse anti-human IFN$\gamma$ antibody, in $100 \mu \mathrm{L}$ volumes of culture medium per well. The cells were left unstimulated (negative control), or were stimulated with $50 \mu \mathrm{L}$ phytohaemagglutinin (positive control) or $50 \mu \mathrm{L}$ of ESAT- 6 and CFP-10 peptides in separate wells. Culture of the plates, washing, counterstaining, visualisation and analysis of the spots were performed according to the manufacturer's recommendations.

The response of stimulated cultures was considered positive when the test well contained at least six more spots and had twice the number of spots than the control well. The background number of spots in negative control wells was always $<10$ spots per well.

\section{MTB-specific nucleic acid technique amplification}

MTB-specific nucleic acid technique amplification (MTB-NAT) was performed by either the BDProbeTec ET assay (Becton Dickinson, Sparks, MD, USA) or the Amplified MTD (GenProbe, San Diego, CA, USA).

\section{Statistical analysis}

All statistical tests were performed as exploratory analyses without adjustment for multiple testing, with nominal significance defined as $p<0.05$. Continuous variables were compared using nonparametric testing (Mann-Whitney U-test).

\section{RESULTS}

Eight patients (four female, four male) with a median age of 43 yrs and with one-sided exudative PE had a definitive diagnosis of plTB (table 1). Two additional patients (aged 43 and $22 \mathrm{yrs}$ ) were confirmed to have plTB by a positive MTBspecific nucleic acid amplification on PE/pleural biopsy in the absence of a positive MTB culture. The total number of confirmed cases of tuberculous pleurisy was therefore 10 . Another 10 patients with a median age of 34 yrs (one female, nine males) with one-sided exudative PE had a presumptive diagnosis of plTB. In total, 21 patients (six females, 15 males), with a median age of $69 \mathrm{yrs}$, had one-sided exudative PE of other origin than TB and were included in the study as controls. These controls were older than patients with confirmed or presumed TB $(\mathrm{p}<0.005)$. Nine of the controls had exudative pleurisy post-pneumonia, eight had malignant PE (nonsmall cell lung cancer, $n=4$; mesothelioma, $n=2$; lymphoma, $\mathrm{n}=1$; breast cancer, $\mathrm{n}=1$ ), three had PE associated with fibrotic-fibroblastic pleurisy, and one had PE associated with rheumatoid arthritis. 


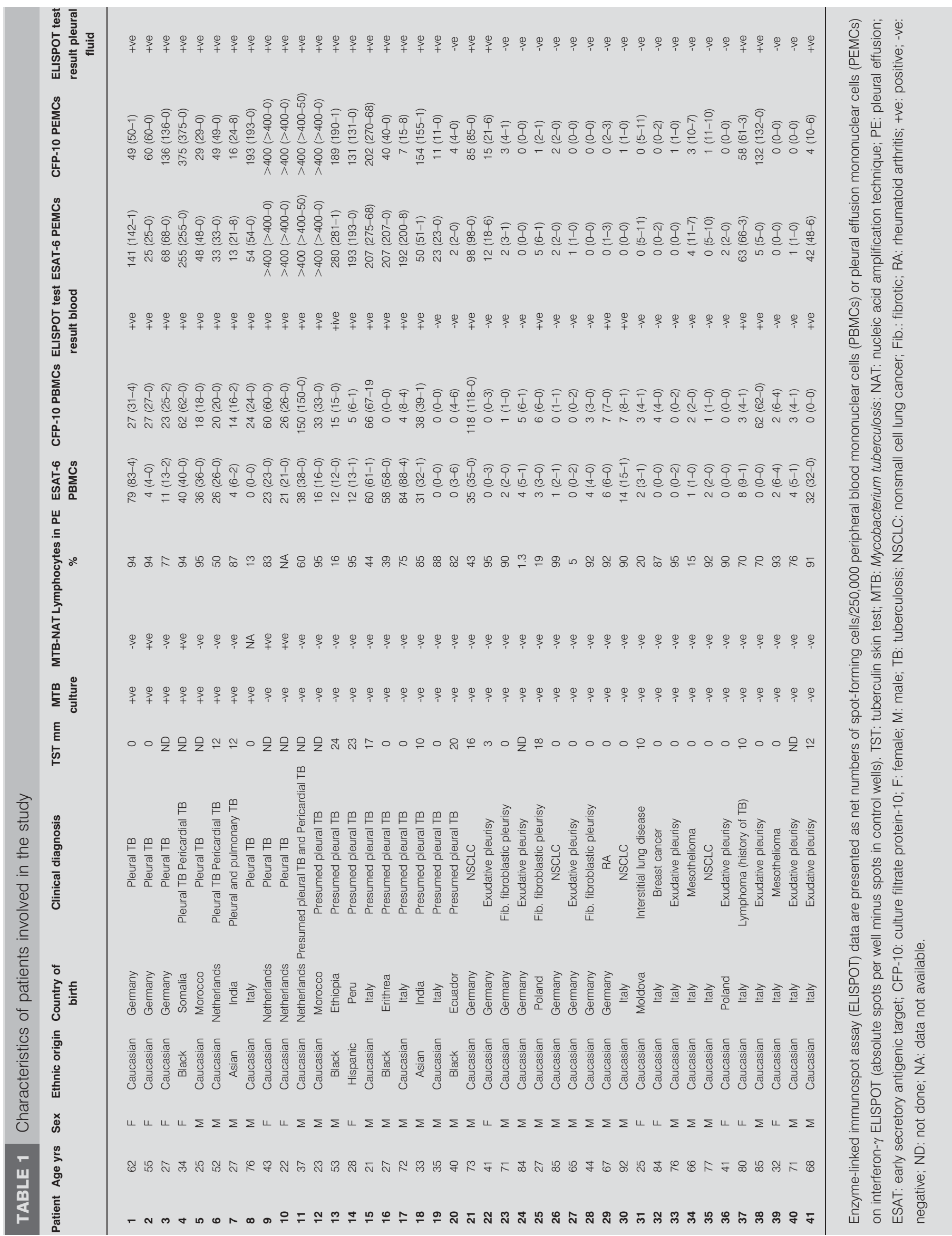


The median proportion of lymphocytes in PEs was $87 \%$ (range $13-95 \%)$ in the group of patients with confirmed plTB, 79\% (range 16-95\%) in the group of patients with presumptive plTB (combined median proportion of $83 \%$ in both TB groups) and $90 \%$ (range 1.3-99\%) in the control group. The proportion of lymphocytes was not statistically different among all three groups.

In all 10 patients with confirmed plTB, the results of $\mathrm{T}$ SPOT.TB were positive $(100 \%)$ in both PBMCs (median number of spot-forming cells (SFCs) was 22 and 25 out of 250,000 for ESAT-6 and CFP-10, respectively) and PEMCs (median number of SFCs was 61 and 98 out of 250,000 for ESAT-6 and CFP-10, respectively; fig. 1). Among the 10 patients with a presumptive diagnosis of plTB, T-SPOT.TB results was positive in eight $(80 \%)$ when performed on PBMCs (median number of SFCs was 24 and 10 out of 250,000 for ESAT-6 and CFP-10, respectively) and in nine (90\%) when performed on PEMCs (median number of SFCs was 200 and 143 out of 250,000 for ESAT-6 and CFP-10, respectively). Among the 21 control patients, T-SPOT.TB was positive in seven $(33 \%)$ when performed on PBMCs (median number of SFCs was two and three out of 250,000 for ESAT-6 and CFP-10, respectively) and in five (24\%) when performed on PEMCs (median number of SFCs was one out of 250,000 for ESAT-6 and CFP-10). The numbers of SFCs in PBMC or PEMC samples were significantly different between controls and patients with both confirmed $(p<0.001)$ and presumptive $(p<0.001)$ plTB. No significant differences were observed between patients with confirmed or presumptive plTB. ESAT-6- and CFP-10specific cells were more highly concentrated in the pleural fluid when compared with the peripheral blood by factors of 6.2 and 4.2 in patients with confirmed plTB and factors of 7.0 and 7.5 in patients with presumptive plTB $(p<0.001)$. The sensitivity and specificity of T-SPOT.TB for the diagnosis of plTB was 90 and $67 \%$ when performed on PBMCs, and 95 and $76 \%$ when performed on PEMCs, respectively. The size of the TST induration did not correlate with numbers of ESAT-6 or CFP-10 SFC.

\section{Discussion}

Cell-mediated immunity plays a key role in the host defence against MTB infection [31-33].

While only a small percentage of T-lymphocytes are found among PBMCs, antigen-specific effector memory T-lymphocytes migrate to the site of inflammation in active TB and rapidly release $\mathrm{T}$-helper cell type- 1 cytokines upon contact with antigens [34-37]. In plTB, the concentrations of $\mathrm{T}$ lymphocytes are significantly higher in pleural fluid than in peripheral blood, while this difference is not observed in patients with nontuberculous PE [38]. In plTB, in vitro simulation of lymphocytes with purified protein derivate leads to T-cell proliferation and release of pro-inflammatory cytokines, including IFN- $\gamma$ [34]. For the diagnosis of TB from blood, ex vivo MTB-specific T-cell IFN- $\gamma$ release assays with RD-1 encoded antigens ESAT-6 and CFP-10 have been developed for commercial use both as ELISPOT and ELISA. While the diagnostic sensitivity and specificity for active TB of both tests is higher compared with the TST [28-30, 39-42], neither test can discriminate between active TB and LTBI when performed on blood [43, 44].
However, recently it was demonstrated that enumeration of MTB-specific mononuclear cells from the site of the infection by ELISPOT can distinguish between active TB, LTBI or other diseases with a high diagnostic sensitivity and specificity [25, 26]. In smear-negative pulmonary $\mathrm{TB}$, the mean numbers of ESAT-6 and CFP-10 SFCs in lung mononuclear cells were 9.6and 7.9-fold higher than in PBMCs [23]. In a smaller study on plTB where only ESAT-6 antigen was used, the mean number of ESAT-6 SFCs in PE mononuclear cells was 15-fold higher than in PBMCs [24].

In the present study, the possibility of a rapid diagnosis of plTB using a commercially available MTB-specific ELISPOT in a routine clinical practice setting was further evaluated. By enumerating antigen-specific mononuclear cells from the peripheral blood and PE of patients with exudative pleurisy, the diagnostic sensitivity of T-SPOT.TB for active plTB was very high $(95 \%)$. In patients with a final diagnosis of plTB the median concentration of ESAT-6 and CFP-10-specific cells was 4.2- to 7.5-fold higher in PEMCs than in PBMCs, thus suggesting a compartmentalisation of antigen-specific T-cells in plTB. In clinical practice, comparing numbers of MTBspecific T-cells in peripheral blood and PE might therefore be a useful strategy for the differentiation of active tuberculous pleurisy from LTBI rather than counting the absolute number of SFCs in each compartment alone.

In the present multicentre study, only two (25\%) out of eight patients with culture-confirmed plTB had a positive MTB-NAT result and only four (20\%) out of 20 patients with a final diagnosis of plTB (confirmed and presumptive cases) had a positive MTB-NAT result on PE. Previous studies [45, 46] showed that the sensitivity of MTB-NAT is lowest in paucibacillary diseases, in particular plTB [47, 48]. The sensitivity of MTB-NAT performed in single-centre studies on PE for the diagnosis of plTB ranges from $25 \%$ in culturenegative patients to $100 \%$ in culture-positive patients [14, 49].

However, the diagnostic specificity of the T-SPOT.TB test was suboptimal $(76 \%)$, thus nearly one out of four patients had a false-positive test result. A limited specificity is a common problem with diagnostic tests in low incidence settings. Five out of 21 patients without active plTB and an alternative diagnosis for an exudative pleural effusion tested positive with the MTB-specific ELISPOT on PEMCs. Four out of five of these patients were presumed to be latently infected with MTB as a result of the PBMC ELISPOT and were also positive by PEMC ELISPOT. One of these patients was 80-yrs-old and had a history of healed pulmonary TB treated in the past (table 1). At the time of the present study, the patient was suffering from a malignant lymphoma. One patient with a negative PBMC ELISPOT result and a positive PEMC ELISPOT result was identified as a recent contact to an index person with smearpositive pulmonary TB. She presented to the hospital with a 6week history of fever and night sweats and was found to have an exudative pleural effusion. Her TST was negative and the pleural effusion resolved without specific treatment. Nevertheless, it might be hypothesised that this patient indeed had acute TB pleurisy and that an effective adaptive host immune response was able to spontaneously resolve the infection. If this was the case, then the PBMC ELISPOT result would have been false negative. The other three patients in the 


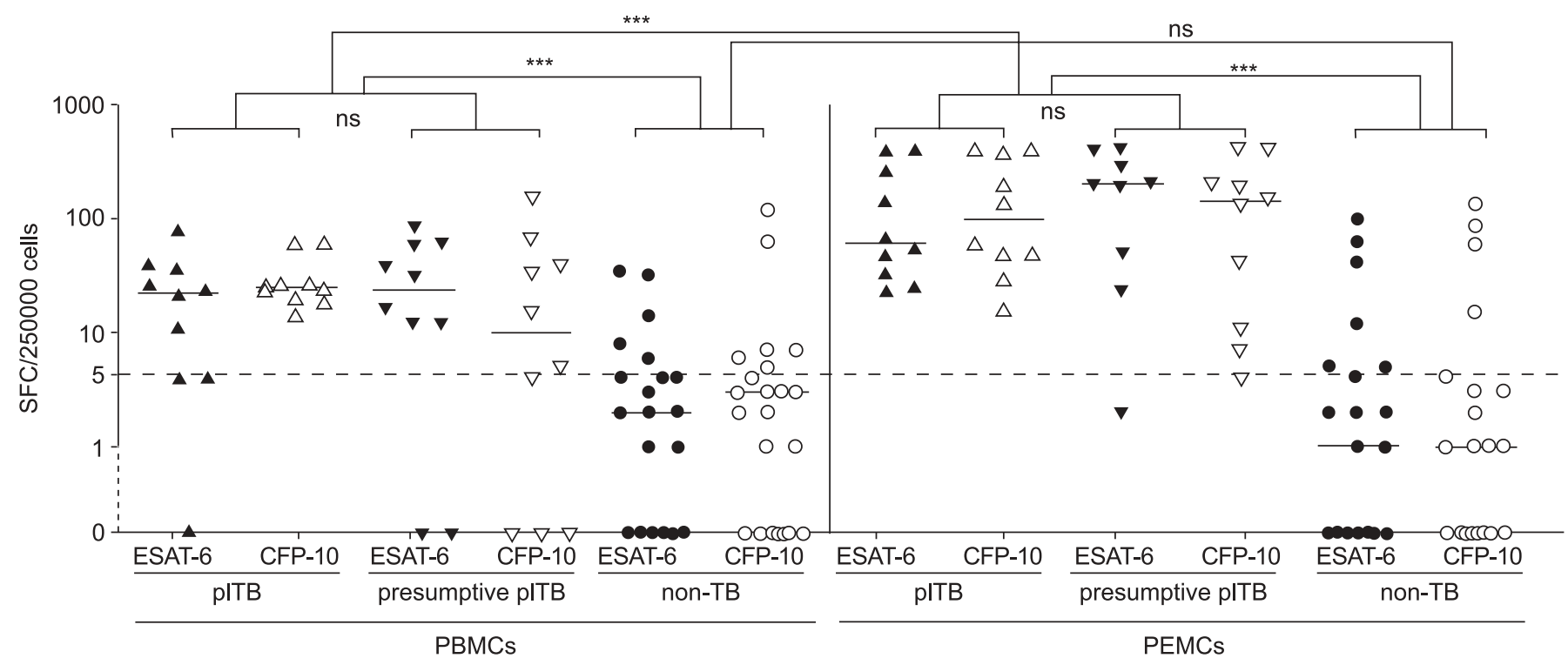

FIGURE 1. Concentration of early secretory antigenic target (ESAT)-6 and culture filtrate protein (CFP)-10 interferon (IFN)- $\gamma$ producing spot-forming cells (SFCs) in peripheral blood mononuclear cells (PBMCs) or pleural effusion mononuclear cells (PEMCs) of patients with definitive pleural tuberculosis (pITB; $n=10, \mathbf{\Lambda}$ and $\triangle$ for ESAT-6 and CFP-10 peptides, respectively), presumptive pITB ( $n=10, \boldsymbol{\nabla}$ and $\nabla$ for ESAT- 6 and CFP-10 peptides, respectively) and of patients with nontuberculous exudative pleural effusion (non-TB; $n=21, \bullet$ and $\bigcirc$ for ESAT-6 and CFP-10 peptides, respectively). ------: cut-off value of five SFCs/250,000 cells. All results have been calculated after subtraction of SFC counts in negative control wells. For group comparison by Mann-Whitney test, sums of ESAT-6 and CFP-10 specific spots were combined. Bars represent median values. ns: not significant. ${ }^{*}$ : not statistically significant; ${ }^{* \star *}: p<0.001$.

control group were all aged $>67$ yrs, and all of them had a LTBI, so an ancient TB infection cannot be excluded.

As the lymphocyte predominance in the pleural fluid did not discriminate between patients with TB and other causes of PE, a positive TIGRA was clearly more informative for the diagnosis of plTB than assessing lymphocyte counts alone. Comparison of results with other diagnostic tests needs to be carried out with caution because of differences in prevalence of active TB in these studies. In the past, several biological markers have been studied to aid the diagnosis of plTB, including the measurement of pleural fluid concentrations of adenosine deaminase $[50,51]$, tumour necrosis factor- $\alpha$ [52-54] and whole IFN- $\gamma[50,55,56]$, with reported diagnostic sensitivities ranging from $72-88 \%$ and specificities from 88 $100 \%$ [50-56]. Thus, reported sensitivities of adenosine deaminase and IFN- $\gamma$ measurements in PE for the diagnosis of plTB are similar to the sensitivity of the T-SPOT.TB test reported in the present study, although T-SPOT.TB specificity seems lower. In future studies, it will be interesting to compare MTB-specific TIGRAs against adenosine deaminase and IFN- $\gamma$ for the diagnosis of plTB.

The present study has three major limitations. First, pleural TB could only be confirmed by culture of MTB or detection of MTB-DNA by NAT in one-half of cases. It is therefore impossible to exclude the fact that patients who fulfilled the clinical diagnosis of presumptive plTB in fact did not have active plTB. However, patients with confirmed plTB and presumptive plTB have been evaluated separately with results in the two groups not statistically different. Moreover, plTB is commonly culture-negative and inclusion of such cases is therefore essential for evaluation of diagnostic tests of improved sensitivity in routine clinical practice. Secondly, no records for the red blood cell content of the PEs exists. In control patients with LTBI, false-positive ELISPOT results on PE may have resulted from contamination with PBMCs from blood. Thirdly, although the present trial is multicentred, the numbers of subjects enrolled from low incidence countries are limited and the results will need to be confirmed in larger cohorts, including immunosuppressed patients and in countries with higher TB incidence [57].

In conclusion, a high diagnostic sensitivity of T-SPOT.TB was found for active pleural tuberculosis when mononuclear cells from pleural effusions were directly evaluated in routine clinical practice in a low tuberculosis incidence setting. The absence of Mycobacterium tuberculosis-specific cells in pleural effusions almost always ruled out a diagnosis of active pleural tuberculosis. Conversely, as specificity of T-SPOT.TB was $\sim 80 \%$, positive test results should always be interpreted with caution and in the full context of the diagnostic work-up of patients with exudative pleural effusion. Large prospective studies of the pleural fluid ELISPOT in patients with suspected pleural tuberculosis are now warranted to determine the true specificity of the assay in high and low prevalence settings; such studies should also investigate whether specificity can be improved if blood-stained pleural effusions are excluded.

\section{ACKNOWLEDGEMENTS}

The authors would like to thank A. Lalvani (Imperial College, London, UK) for his critical review of the manuscript.

\section{REFERENCES}

1 Elzinga G, Raviglione MC, Maher D. Scale up: meeting targets in global tuberculosis control. Lancet 2004; 363: 814-819. 
2 Vidal R, de Gracia J, Ruiz J, Fite E, Monsó E, Martín N. Controlled study of 637 patients with tuberculosis. Diagnosis and therapeutic results with 9- and 6-month regimens. Med Clin (Barc) 1986; 87: 368-370.

3 Valdes L, Pose A, San José E, Martinez Vázquez JM. Tuberculous pleural effusions. Eur J Intern Med 2003; 14: 77-88.

4 Gopi A, Madhaven SM, Sharma SK, Sahn SA. Diagnosis and treatment of tuberculous pleural effusion in 2006. Chest 2007; 131: 880-889.

5 Kumar S, Seshadri MS, Koshi G, John TJ. Diagnosing tuberculous pleural effusion: comparative sensitivity of mycobacterial culture and histopathology. Br Med J (Clin Res Ed) 1981; 283: 20.

6 Prakash UB, Reiman HM. Comparison of needle biopsy with cytologic analysis for the evaluation of pleural effusion: analysis of 414 cases. Mayo Clin Proc 1985; 60: 158-164.

7 Seibert AF, Haynes J Jr, Middleton R, Bass JB Jr. Tuberculous pleural effusion. Twenty-year experience. Chest 1991; 99: 883-886.

8 Valdes L, Alvarez D, San José E, et al. Tuberculous pleurisy: a study of 254 patients. Arch Intern Med 1998; 158: 2017-2021.

9 Berger HW, Mejia E. Tuberculous pleurisy. Chest 1973; 63: 88-92.

10 Epstein DM, Kline LR, Albelda SM, Miller WT. Tuberculous pleural effusions. Chest 1987; 91: 106-109.

11 Escudero Bueno C, Garca Clemente M, Cuesta Castro B, et al. Cytologic and bacteriologic analysis of fluid and pleural biopsy specimens with Cope's needle. Study of 414 patients. Arch Intern Med 1990; 150: 1190-1194.

12 Aggarwal AN, Gupta D, Jindal SK. Diagnosis of tuberculous pleural effusion. Indian J Chest Dis Allied Sci 1999; 41: 89-100.

13 Sibley JC. A study of 200 cases of tuberculous pleurisy with effusion. Am Rev Tuberc 1950; 62: 314-323.

14 Nagesh BS, Sehgal S, Jingal SK, Arora SK. Evaluation of polymerase chain reaction for detection of Mycobacterium tuberculosis in pleural fluid. Chest 2001; 119: 1737-1741.

15 Richeldi L. Rapid identification of Mycobacterium tuberculosis infection. Clin Microbiol Infect 2006; 12: Suppl. 9, 34-36.

16 Richeldi L, Ewer K, Losi M, et al. Early diagnosis of subclinical multidrug-resistant tuberculosis. Ann Intern Med 2004; 140: 709-713.

17 Pai M, Riley LW, Colford JM Jr. Interferon-gamma assays in the immunodiagnosis of tuberculosis: a systematic review. Lancet Infect Dis 2004; 4: 761-776.

18 Gooding S, Gooding S, Chowdhury O, Hinks T. Impact of a T cell-based blood test for tuberculosis infection on clinical decisionmaking in routine practice. J Infect 2007; 54: e169-e174.

19 Richeldi L, Luppi M, Losi M, et al. Diagnosis of occult tuberculosis in hematological malignancy by enumeration of antigen-specific T cells. Leukemia 2006; 20: 379-381.

20 Liebeschuetz S, Bamber S, Ewer K, Deeks J, Pathan AA, Lalvani A. Diagnosis of tuberculosis in South African children with a T-cell-based assay: a prospective cohort study. Lancet 2004; 364: 2196-2203.

21 Westermann J, Pabst R. Distribution of lymphocyte subsets and natural killer cells in the human body. Clin Investig 1992; 70: 539-544.
22 Maskell NA, Butland RJ. BTS guidelines for the investigation of a unilateral pleural effusion in adults. Thorax 2003; 58: Suppl. 2, ii8-ii17.

23 Schwander SK, Torres M, Sada E, et al. Enhanced responses to Mycobacterium tuberculosis antigens by human alveolar lymphocytes during active pulmonary tuberculosis. J Infect Dis 1998; 178: 1434-1445.

24 Hirsch CS, Toossi Z, Johnson JL, et al. Augmentation of apoptosis and interferon- $\gamma$ production at sites of active Mycobacterium tuberculosis infection in human tuberculosis. J Infect Dis 2001; 183: 779-788.

25 Jafari C, Ernst M, Kalsdorf B, et al. Rapid diagnosis of smear-negative tuberculosis by bronchoalveolar lavage enzyme-linked immunospot. Am J Respir Crit Care Med 2006; 174: 1048-1054.

26 Wilkinson KA, Wilkinson RJ, Pathan A, et al. Ex vivo characterization of early secretory antigenic target 6specific $\mathrm{T}$ cells at sites of active disease in pleural tuberculosis. Clin Infect Dis 2005; 40: 184-187.

27 Davies CW, Gleeson FV, Davies RJ. BTS guidelines for the management of pleural infection. Thorax 2003; 58: Suppl. 2, ii18-ii28.

28 Lalvani A, Pathan AA, Durkan H, et al. Enhanced contact tracing and spatial tracking of Mycobacterium tuberculosis infection by enumeration of antigen-specific T cells. Lancet 2001; 357: 2017-2021.

29 Lalvani A, Pathan AA, McShane H, et al. Rapid detection of Mycobacterium tuberculosis infection by enumeration of antigen-specific T cells. Am J Respir Crit Care Med 2001; 163: 824-828.

30 Lalvani A, Magvenkar P, Udwadia Z, et al. Enumeration of $\mathrm{T}$ cells specific for RD1-encoded antigens suggests a high prevalence of latent Mycobacterium tuberculosis infection in healthy urban Indians. J Infect Dis 2001; 183: 469-477.

31 Tully G, Kortsik C, Höhn H, et al. Highly focused T cell responses in latent human pulmonary Mycobacterium tuberculosis infection. J Immunol 2005; 174: 2174-2184.

32 Chan J, Flynn J. The immunological aspects of latency in tuberculosis. Clin Immunol 2004; 110: 2-12.

33 Kaufmann SH. How can immunology contribute to the control of tuberculosis? Nat Rev Immunol 2001; 1: 20-30.

34 Barnes PF, Lu S, Abrams JS, Wang E, Yamamura M, Modlin RL. Cytokine production at the site of disease in human tuberculosis. Infect Immun 1993; 61: 3482-3489.

35 Okamoto M, Hasegawa Y, Hara T, et al. T-helper type 1/Thelper type 2 balance in malignant pleural effusions compared to tuberculous pleural effusions. Chest 2005; 128: 4030-4035.

36 Hiraki A, Aoe K, Matsuo K, et al. Simultaneous measurement of T-helper 1 cytokines in tuberculous pleural effusion. Int J Tuberc Lung Dis 2003; 7: 1172-1177.

37 Dieli F, Friscia G, Di Sano C, et al. Sequestration of T lymphocytes to body fluids in tuberculosis: reversal of anergy following chemotherapy. J Infect Dis 1999; 180: 225-228.

38 Chen YM, Yang WK, Whang-Peng J, Tsai CM, Perng RP. An analysis of cytokine status in the serum and effusions of patients with tuberculous and lung cancer. Lung Cancer 2001; 31: 25-30.

39 Ferrara G, Losi M, Meacci M, et al. Routine hospital use of a new commercial whole blood interferon-gamma assay for 
the diagnosis of tuberculosis infection. Am J Respir Crit Care Med 2005; 172: 631-635.

40 Ferrara G, Losi M, D'Amico R, et al. Use in routine clinical practice of two commercial blood tests for diagnosis of infection with Mycobacterium tuberculosis: a prospective study. Lancet 2006; 367: 1328-1334.

41 Kang YA, Lee HW, Yoon HI, et al. Discrepancy between the tuberculin skin test and the whole-blood interferon gamma assay for the diagnosis of latent tuberculosis infection in an intermediate tuberculosis-burden country. JAMA 2005; 293: 2756-2761.

42 Ravn P, Munk ME, Andersen AB, et al. Prospective evaluation of a whole-blood test using Mycobacterium tuberculosis-specific antigens ESAT-6 and CFP-10 for diagnosis of active tuberculosis. Clin Diagn Lab Immunol 2005; 12: 491-496.

43 Richeldi L. An update on the diagnosis of tuberculosis infection. Am J Respir Crit Care Med 2006; 174: 736-742.

44 Menzies D, Pai M, Comstock G. Meta-analysis: new tests for the diagnosis of latent tuberculosis infection: areas of uncertainty and recommendations for research. Ann Intern Med 2007; 146: 340-354.

45 American Thoracic Society, Rapid diagnostic tests for tuberculosis: what is the appropriate use? American Thoracic Society Workshop. Am J Respir Crit Care Med 1997; 155: 1804-1814.

46 Chakravorty S, Sen MK, Tyagi JS. Diagnosis of extrapulmonary tuberculosis by smear, culture, and PCR using universal sample processing technology. J Clin Microbiol 2005; 43: 4357-4362.

47 Villegas MV, Labrada LA, Saravia NG. Evaluation of polymerase chain reaction, adenosine deaminase, and interferon- $\gamma$ in pleural fluid for the differential diagnosis of pleural tuberculosis. Chest 2000; 118: 1355-1364.

48 Lima DM, Colares JK, da Fonseca BA. Combined use of the polymerase chain reaction and detection of adenosine deaminase activity on pleural fluid improves the rate of diagnosis of pleural tuberculosis. Chest 2003; 124: 909-914.

49 Kim SY, Park YJ, Kang SJ, Kim BK, Kang CS. Comparison of the BDProbeTec ET system with the Roche COBAS AMPLICOR System for detection of Mycobacterium tuberculosis complex in the respiratory and pleural fluid specimens. Diagn Microbiol Infect Dis 2004; 49: 13-18.

50 Hiraki A, Aoe K, Eda R, et al. Comparison of six biological markers for the diagnosis of tuberculous pleuritis. Chest 2004; 125: 987-989.

51 Aoe K, Hiraki A, Murakami T, et al. Diagnostic significance of interferon- $\gamma$ in tuberculous pleural effusions. Chest 2003; 123: 740-744.

52 Tahhan M, Ugurman F, Gozu A, Akkalyoncu B, Samurkasoglu B. Tumour necrosis factor- $\alpha$ in comparison to adenosine deaminase in tuberculous pleuritis. Respiration 2003; 70: 270-274.

53 Wong CF, Yew WW, Leung SK, et al. Assay of pleural fluid interleukin-6, tumour necrosis factor- $\alpha$ and interferon- $\gamma$ in the diagnosis and outcome correlation of tuberculous effusion. Respir Med 2003; 97: 1289-1295.

54 Ogawa K, Koga H, Hirakata Y, Tomono K, Tashiro T, Kohno S. Differential diagnosis of tuberculous pleurisy by measurement of cytokine concentrations in pleural effusion. Tuber Lung Dis 1997; 78: 29-34.

55 Wongtim S, Silachamroon U, Ruxungtham K, et al. Interferon- $\gamma$ for diagnosing tuberculous pleural effusions. Thorax 1999; 54: 921-924.

56 Villena V, López-Encuentra A, Pozo F, et al. Interferon- $\gamma$ levels in pleural fluid for the diagnosis of tuberculosis. Am J Med 2003; 115: 365-370.

57 Lange C, Hellmich B, Ernst M, Ehlers S. Rapid immunodiagnosis of tuberculosis in a woman receiving anti-TNF therapy. Nat Clin Pract Rheumatol 2007; 3: 528-534. 